\title{
Avaliação das Condições Higiênico-Sanitárias e Físico-Estruturais em Unidades de Alimentação e Nutrição de um Estado do Nordeste Brasileiro
}

\section{Evaluation of Hygienic-Sanitary and Physical-Structural Conditions of Food and Nutrition Units in a Northeastern Brazilian State}

\author{
Kaira Cristina Ferreira Araújo Rebouçasa; Maria Madalena Oliveira Jorge ${ }^{\mathrm{a}}$; Eliakim Aureliano da Silva ${ }^{\mathrm{b}}$; Beatriz \\ Gonçalves Feitosa dos Santos ${ }^{\mathrm{b}}$; Cláudia Lorena Ribeiro Lopes ${ }^{\mathrm{b}}$; Gleyson Moura dos Santos ${ }^{\mathrm{b}}$; Nara Vanessa dos Anjos \\ Barros*b; Daila Leite Chaves Bezerra ${ }^{\mathrm{b}}$; Ednelda Brito Machado ; Paulo Víctor de Lima Sousa ${ }^{\mathrm{a}}$
}

\author{
${ }^{a}$ Centro Universitário Maurício de Nassau. PI, Brasil. \\ bUniversidade Federal do Piauí. PI, Brasil. \\ *E-mail: nara.vanessa@hotmail.com
}

\begin{abstract}
Resumo
Nas Unidades de Alimentação e Nutrição (UAN), as contaminações podem ser oriundas das diversas etapas de manuseio do alimento, aumentando, assim, a incidência de doenças de origem alimentar, sendo necessária a manutenção de condições higiênico-sanitárias adequadas e locais apropriados, de forma a garantir a dignidade do ato de se alimentar. O presente trabalho teve como objetivo analisar as condições higiênico-sanitárias e físico-estruturais de Unidades de Alimentação e Nutrição. O estudo foi realizado em UAN dos municípios de Teresina, Picos, Floriano e Parnaíba no Estado do Piauí, nas quais foram codificadas em A, B, C, D, E, F e G. Realizou-se a aplicação do checklist adaptado da RDC no 275/2002, em que cada item foi avaliado em "conforme" e "não-conforme" e seus resultados foram apresentados em porcentagens. Das 7 UAN analisadas, 57\% eram UAN institucionais, 29\% UAN hospitalares e 14\% UAN não institucionais, a maioria apresentou percentuais de conformidades maior que $70 \%$, destacando a UAN A $(95,3 \%)$. A UAN E foi a que apresentou menor percentual de adequação na maioria dos itens, destacando o item matérias-primas, ingredientes e embalagens (18,2\%), sendo classificada como muito ruim. A UAN G foi a única que não apresentou documentos e registro. Conclui-se que a maioria das UAN apresentou condições higiênico-sanitárias satisfatórias em boa parte dos itens preconizados pela RDC n 216/2004. Entretanto, algumas UAN foram classificadas como ruins, podendo gerar riscos à saúde dos comensais.
\end{abstract}

Palavras-chave: Alimentação Coletiva. Segurança Alimentar e Nutricional. Manipulação de Alimentos. Boas Práticas de Fabricação.

\begin{abstract}
In Food and Nutrition Units (UAN), contamination may come from the various stages of food handling, thus increasing the incidence of foodborne illnesses, requiring the maintenance of adequate hygienic-sanitary conditions and appropriate places, to guarantee the dignity of the feeding act. The study was carried out in UAN in the municipalities of Teresina, Picos, Floriano and Parnaiba in the state of Piaui, in which they were coded in $A, B, C, D, E, F$ and $G$. The checklist adapted from RDC $n^{\circ} 275 / 2002$ was applied, in which each item was evaluated as "compliant" and "non-compliant" and its results were presented in percentages. Of the 7 UANs analyzed, 57\% were institutional UANs, 29\% hospital UANs and 14\% non-institutional UANs, the majority had conformity percentages greater than 70\%, highlighting UAN A (95.3\%). The UAN E was the one with the lowest percentage of adequacy in most items, highlighting the item raw materials, ingredients and packaging $(18.2 \%)$, being classified as very bad. The UAN G was the only one that did not present documents and registration. It is concluded that the majority of UANs presented satisfactory hygienic-sanitary conditions in most of the items recommended by RDC $n^{\circ} 216 / 2004$. However, some UANs have been classified as bad, which can create health risks for diners.
\end{abstract}

Keywords: Collective Food. Food and Nutrition Security. Food Handling. Good Manufacturing Practices.

\section{Introdução}

Os processos de industrialização, de globalização e de urbanização geraram mudanças de comportamento da população, em que se destaca a realização da alimentação fora do domicílio. No Brasil, o crescimento do percentual do gasto com a alimentação fora do lar demonstra essa tendência que vem se popularizando (VAZ; BENNEMANN, 2018).

A partir disso, o setor de alimentação coletiva tem se expandido, significativamente, no Brasil, com uma estimativa de 14,7 milhões de refeições servidas diariamente e uma receita de cerca de 23,1 bilhões de reais anuais entre impostos e contribuições em 2020 (ABERC, 2020). Isto expressa, de um lado, a importância e a expansão da alimentação coletiva no Brasil e, de outro, o comprometimento que se deve ter para com a qualidade da alimentação produzida por esses estabelecimentos (BOFF; STRASBURG, 2018).

Nessa perspectiva, a procura por refeições prontas para o consumo fomentou o mercado da alimentação coletiva e diversificou seus serviços, com destaque para o aumento no número de Unidades de Alimentação e Nutrição (UAN), que são serviços produtores de alimentação para coletividades, que possuem um conjunto de áreas definidas, organizadas em sequências, com fornecimento de refeições balanceadas (OLIVEIRA; LIBOREDO, 2018). As UAN devem possuir, além dos padrões dietéticos, os higiênicos que devem estar ajustados às condições financeiras do local, no intuito final 
de atender às necessidades nutricionais de seus comensais (BEZERRA et al. 2017; VAZ; BENNEMANN, 2018).

O controle das condições higiênico-sanitárias nos locais em que os alimentos são manipulados é importante, uma vez que contaminações podem ocorrer nas diversas etapas do preparo do alimento. Para tanto, a atenção acerca da estrutura física das UAN se torna primordial, pois é o local que comporta todos os procedimentos envolvidos na produção de alimentos. Nesse cenário, surgem as resoluções normativas que dispõem sobre o regulamento técnico em produção de alimentos (MACIEL et al., 2017; SANTOS et al., 2019).

O planejamento da estrutura física de uma UAN permite analisar as necessidades específicas do estabelecimento para que o funcionamento aconteça, de forma adequada, com uma linha de produção coerente, sem ociosidade de equipamentos por má localização, sem condições de trabalho estressantes, sem inadequações na iluminação, ventilação ou na temperatura, proporcionando uma qualidade de trabalho satisfatória com um produto final com qualidade higiênicosanitária sem custos elevados e com segurança alimentar e nutricional (SANTOS et al., 2019).

As falhas nos setores de manipulação da UAN decorrentes de espaço físico inadequado e a ausência de fiscalização rigorosa são as principais causas das ocorrências de Doenças Transmitidas por Alimentos (DTA). Esta, por sua vez, configura um problema persistente, que continua a preocupar a população mundial em função da grande demanda nos serviços de alimentação (MENDES et al, 2019; BOFF; STRASBURG, 2018). No Brasil, no período de 2009 a 2018 , foram registrados 6.809 episódios de DTA, com 634.568 pessoas expostas, 120.584 doentes, 16.632 hospitalizados, 99 óbitos e uma taxa de letalidade de $0,08 \%$ o que gera preocupação para a população mundial. Essas doenças são os resultados do consumo de fontes alimentares contaminadas por agentes químicos, físicos ou biológicos (BRASIL, 2018).

Com isso, torna-se imprescindível que as UAN estejam adequadas, tanto nos aspectos higiênicos quanto estruturais, para garantir o fornecimento de uma alimentação segura (OLIVEIRA; LIBOREDO, 2018). Diante disso, o presente estudo teve como objetivo avaliar as condições higiênicosanitárias e físico-estruturais de unidades de alimentação e nutrição de um Estado do Nordeste brasileiro.

\section{Material e Métodos}

Trata-se de uma pesquisa de corte transversal, exploratória, descritiva e quantitativa que foi realizado em UAN de Instituições de Ensino Superior, de hospitais e em restaurante popular localizadas nos municípios de Floriano, Parnaíba, Picos e Teresina do Estado do Piauí.

Para a coleta dos dados, foi solicitada a autorização institucional para concessão das informações nas UAN, declarando que serão mantidas em sigilo as fontes dos dados. Com isso, as UAN foram codificadas em A, B, C, D, E, F e
G, a fim de manter o anonimato das instituições em questão.

Para a avaliação das condições higiênico-sanitárias e físicoestruturais das UAN, foi utilizado o checklist adaptado da RDC n ${ }^{\circ} 275$ de 21 de outubro de 2002, do Ministério da Saúde, que dispõe sobre o Regulamento Técnico de Procedimentos Operacionais Padronizados aplicados aos Estabelecimentos Produtores/Industrializadores de Alimentos (BRASIL, 2002), que tem como base a Resolução RDC no 216/2004 da Agência Nacional de Vigilância Sanitária (BRASIL, 2004), na qual dispõe sobre o Regulamento Técnico de Boas Práticas para Serviços de Alimentação.

A lista de verificação possuía um total de 157 itens, que foram organizados em 8 (oito) blocos temáticos: I- Edificações e instalações; II- Equipamentos, móveis e utensílios; III- Manipuladores; IV- Matérias-primas, Ingredientes e Embalagens; V- Preparação do Alimento; VIArmazenamento; VII- Exposição do consumo do alimento e VIII- Documentação.

Posteriormente, cada item foi avaliado e classificado em "adequado" (AD), quando este se encontra em acordo com a RDC no 216/2004, “inadequado" (IN) quando não está em conformidade e "não aplicável" (NA), quando não é aplicável à realidade do local. Os itens considerados "não aplicável" foram subtraídos no cálculo da porcentagem de adequação.

Para o cálculo das médias de adequação foi realizada uma regra de três simples, utilizando-se a seguinte fórmula:

$\%$ Adequação Total $=\frac{(\text { itens avaliados }- \text { itens não aplicáveis })}{\text { itens adequados }}=\frac{100 \%}{X}$

Posteriormente, as UAN foram classificadas, segundo Saccol, Stangarlin e Hecktheuer (2013), quanto à adequação dos requisitos em muito boa (91 a 100\%), boa (70 a 90\%), regular (50 a 69\%), ruim (20 a 49\%) e muito ruim (0 a 19\%).

Após a coleta das informações, o programa Microsoft ${ }^{\circledR}$ Excel, versão 2014 foi utilizado para análise dos dados e para obtenção da frequência dos itens conformes e não conformes, segundo a RDC no 216/2004. Em seguida, os resultados foram apresentados em forma de gráficos e tabelas em valores percentuais.

\section{Resultados e Discussão}

Foram coletados dados de um total de 7 UAN, sendo 57\% UAN institucionais $(n=4), 29 \%$ UAN hospitalares $(n=2)$ e $14 \%$ UAN não institucionais $(n=1)$. As UAN institucionais e não institucionais possuem horário de funcionamento de segunda ao sábado, das 06h00min às $07 \mathrm{~h} 30 \mathrm{~min}$ (café da manhã), 11h00min às 13h30min (almoço) e 17h00min às 19h00min (jantar), com uma média de distribuição de 2.500 refeições por dia. Já as UAN hospitalares funcionam, diariamente, nos mesmos horários citados anteriormente, com média de 500 refeições por dia. O sistema de distribuição das refeições de todas as UAN é tipo centralizado, bem como possuem nutricionistas como responsáveis técnicos pelo serviço. A Figura 1 demonstra o percentual de conformidades nas UAN avaliadas. 
Figura 1 - Percentual geral de conformidade das Unidades de Alimentação e Nutrição (UAN)

120,0

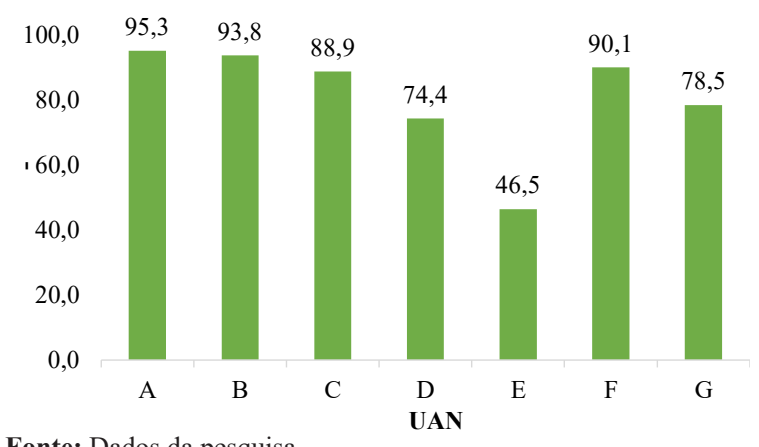

Fonte: Dados da pesquisa.

Das 7 UAN avaliadas, 6 apresentaram percentuais de conformidade maior do que $70 \%$ (A, B, C, D, F e G), sendo consideradas adequadas, com destaque paraaUANinstitucional A, que obteve o maior percentual de conformidade $(95,3 \%)$, resultado este considerado satisfatório, pois um ambiente adequado pode garantir à saúde dos usuários dos serviços de alimentação. Esse elevado percentual de conformidade decorre da presença de um fluxo de produção adequado, o que pode minimizar os riscos de contaminação. Entretanto, a UAN não institucional E apresentou um percentual de 46,5\% de conformidade, sendo classificada como ruim.

Dare, Bernardo e Chaud (2018) avaliaram as condições físicas e sanitárias de uma UAN hospitalar localizada no município de São Paulo e verificaram resultados semelhantes ao do presente estudo, com um percentual 92,3\% de conformidade. Locateli e Silva (2017) também apresentaram resultados satisfatório para as UAN de Hotelaria e do Programa de Alimentação do Trabalhador (PAT), que obtiveram um percentual de $88,3 \%$ e $74,2 \%$ de conformidade, respectivamente.

Com a aplicação da Lista de Avaliação de Boas Práticas para Serviços de Alimentação é capaz averiguar o grau de adequação às Boas Práticas, conforme RDC n 216/2004 da ANVISA (BRASIL, 2004), sendo este um instrumento que deve ser utilizado para corrigir as possíveis inadequações para garantir a produção de uma alimentação segura para a saúde dos comensais. A Tabela 1 apresenta o percentual de conformidade por item avaliados na UAN, segundo a RDC $n^{\circ} 216 / 2004$.

Quadro 1 - Percentual de conformidade por item avaliado das Unidades de Alimentação e Nutrição (UAN)

\begin{tabular}{|l|c|c|c|c|c|c|c|}
\hline \multicolumn{1}{|c|}{ Itens Avaliados } & \multicolumn{5}{c|}{ UAN (\%) } \\
\cline { 2 - 8 } & A & B & C & D & E & F & G \\
\hline 1. Edificações e Instalações & 86,5 & 96,0 & 76,0 & 63,3 & 32,2 & 58,8 & 77,5 \\
\hline 2. Equipamentos, Móveis e Utensílios & 100 & 100 & 87,5 & 60,3 & 46,7 & 85,7 & 95,3 \\
\hline 3. Manipuladores & 92,9 & 81,1 & 72,7 & 100 & 98,2 & 85,7 & 85,7 \\
\hline 4. Matérias-primas, Ingredientes e Embalagens & 100 & 100 & 90,9 & 81,8 & 18,2 & 100 & 100 \\
\hline 5. Preparação do Alimento & 83,3 & 83,3 & 83,9 & 70,0 & 53,3 & 90,9 & 69,7 \\
\hline 6. Armazenamento & 100 & 100 & 100 & 50,0 & 33,3 & 100 & 100 \\
\hline 7. Exposição do consumo do alimento & 100 & 90,0 & 100 & 70,0 & 40,0 & 100 & 100 \\
\hline 8. Documentação e Registro & 100 & 100 & 100 & 100 & 50,0 & 100 & 0,0 \\
\hline
\end{tabular}

A UAN que apresentou menor percentual de conformidade na maioria dos itens avaliados foi a $\mathrm{E}$, fato este que pode gerar comprometimento e problemas no desempenho no fluxo de trabalho dentro dessa UAN não institucional. As demais UAN apresentaram percentuais mais elevados para a maioria dos itens avaliados, sendo estas consideradas mais adequadas.

Para o item edificações e instalações, a UAN hospitalar B apresentou maior percentual de adequação (96\%), sendo classificada como muito boa. Já a UAN E, apresentou 32\% de conformidade para este item, sendo classificada como ruim. Este baixo percentual decorre de a mesma apresentar condições precárias, a área interna do ambiente apresenta elevado número de objetos em desuso, o piso não é de revestimento liso e permeável, no qual dificulta sua lavagem, o teto se encontra em um estado inadequado de conservação com presença de fissuras, de rachaduras, de infiltrações e de goteiras dentro do setor de alimentação.

Segundo a RDC no 216/2004, recomenda-se que as instalações físicas como piso, parede e teto possuam revestimento liso, impermeável e lavável, devem ser mantidos íntegros, conservados, livres de rachaduras, trincas, goteiras, vazamentos, infiltrações, bolores, descascamentos, entre outros e que não devem transmitir contaminantes aos alimentos (BRASIL, 2004).

Com relação aos equipamentos, móveis e utensílios, observou-se que entre todas as UAN analisadas, as UAN A e B apresentaram $100 \%$ de conformidade (Tabela 1 ), visto que possuíam equipamentos e utensílios em um estado de conservação adequado, representando ponto positivo para o funcionamento dessas UAN. Estudos realizado por Dare, Bernardo e Chaud (2018) e Locateli e Silva (2018) também demonstraram resultados positivos para o item em questão, com $94,1 \%$ e $100 \%$ de conformidade, respectivamente, uma vez que as UAN analisadas possuíam planilhas de registro da frequência de higienização com assinaturas dos responsáveis por essa função, os funcionários responsáveis pela higienização das instalações possuíam uniformes apropriados e que utilizavam produtos inodoros nas áreas de produção e 
de armazenamento.

Entre os fatores que justifiquem o menor percentual observado na UAN E (46,7\%) para o item em questão, pode-se destacar o estado de conservação inadequado os equipamentos, os móveis e os utensílios, a presença de corrosões, não havia manutenção periódica e calibração dos equipamentos de medição, bem como não apresentava o registro dessas operações. Além disso, foi observado o armazenamento incorreto de produtos saneantes, o que pode ser uma possível fonte de contaminação cruzada.

Gomes et al. (2012) avaliaram alguns aspectos higiênicosanitários na elaboração de refeições em escolas públicas do Estado de Goiás, nas quais se observaram $82 \%$ de adequações referente ao item equipamentos, móveis e utensílios. Grande parte das inadequações do referido estudo estava relacionadas ao armazenamento de utensílios em espaço inapropriado, como observado nas UAN da presente pesquisa.

No item manipuladores, os percentuais de adequação foram satisfatórios para todas as UAN avaliadas (Quadro 1), com destaque para UAN D (100\%) que, por ser uma UAN hospitalar, há um maior rigor no cumprimento dessas normas, visando o não comprometimento da saúde de seus comensais. Para tanto, este resultado é decorrente de as UAN atenderem as competências referentes às boas práticas dos manipuladores, quanto ao vestuário, aos hábitos higiênicos, o controle de saúde e capacitação desses indivíduos, uma vez que é imprescindível a utilização de uniformes compatíveis com a função e livres de sujidades, sendo de suma relevância também a higienização pessoal.

Reis, Flávio e Guimarães (2015) verificaram dados semelhantes de conformidade para os manipuladores de alimentos, pois eles possuem asseio pessoal adequado, uniformes conservados e limpos, utilizavam os Equipamentos de Proteção Individual (EPI) exigidos, bem como não utilizam adornos (brincos, anéis, pulseiras e outros). A aplicação de estratégias para a manipulação de alimentos e a conscientização dos colaboradores envolvidos são essenciais para o controle das DTA, especialmente, nos estabelecimentos, que fornecem alimentação coletiva.

Dare, Bernardo e Chaud (2018) relataram que apesar dos manipuladores não usarem adornos pessoais, apresentarem barbas aparadas, com cabelos presos e protegidos, era comum que eles conversassem, cantassem e utilizassem o celular dentro da cozinha, sendo esses aspectos considerados como meios de contaminação na manipulação de alimentos e que devem ser evitados, segundo a RDC n 216/2004 (BRASIL, 2004).

No bloco referente às matérias-primas, ingredientes e embalagens, as UAN institucionais A, F e G e a hospitalar B, apresentaram $100 \%$ de conformidade. Em contrapartida, a UAN E apresentou o menor percentual (18,2\%), sendo classificado como muito ruim, visto o que não seguia os critérios preconizados pela RDC nº 216/2004 (BRASIL, 2004). Dare, Bernardo e Chaud (2018) também obtiveram resultados satisfatório para esse bloco (92,3\%), o que representa um ponto positivo para garantir o controle higiênico-sanitário da matéria-prima destinada para a produção de refeições seguras.

Quanto ao item preparação do alimento, a UAN E apresentou, novamente, o menor percentual de adequação $(53,3 \%)$, sendo classificada como regular. Apesar desta UAN ter apresentado alguns cuidados para minimizar os ricos de contaminação, ainda existiam práticas inadequadas ao manipular os alimentos, que devem ser evitadas dentro da UAN, o que repercutiu nos baixos valores percentuais de adequação.

Pittelkow e Bitello (2014) verificaram, em uma UAN, do Sul do país, que a higienização das mãos dos manipuladores era inadequada, pois não faziam o procedimento correto de higienização, seguindo as normas e frequência recomendada, aspecto este também observado no presente estudo.

Para Leão et al. (2018), a higienização adequada das mãos é uma medida essencial para minimizar os riscos de contaminação por micro-organismos, na qual a lavagem deve ser frequente utilizando água corrente, sabão e antisséptico. Lund e O 'Brien et al. (2009) enfatizam que os episódios de surtos envolvendo alimentos contaminados podem ser evitados com a junção de princípios elementares de higiene pessoal e boas práticas.

De acordo com Almeida et al. (2017), no decorrer dos anos, vários regulamentos e normas foram elaborados pelo Governo brasileiro para a área de alimentos. As primeiras focavam a questão higiênica desses, na qual basicamente a presença ou ausência de contaminantes determinava a qualidade do alimento.

A RDC no 2016/2004 recomenda que os funcionários que manipulam os alimentos devem adotar medidas, que reduza os riscos de contaminação e que evitem expor os alimentos em temperaturas capaz de modificar sua matéria-prima, bem como terem cautela ao manusear os alimentos crus, semipreparados e prontos para consumo (BRASIL, 2004).

As UAN A, B, C, F e G apresentarem 100\% de conformidade para o item armazenamento, sendo classificadas como muito boa, já as UAN D e F foram classificadas como regular e ruim, respectivamente. É importante enfatizar que armazenamento dos produtos alimentícios deve ser feito em um local limpo e organizado, identificados e protegidos livres de contaminantes até a distribuição ao consumidor, bem como acondicionado em uma temperatura, que não comprometa a qualidade higiênica-sanitária (BRASIL, 2004).

No item exposição do consumo do alimento, a maioria das UAN apresentou percentuais de conformidade satisfatório, destacando-se as UAN A, C e F, pois essas UAN apresentaram áreas de exposição do alimento preparado limpas com frequente higienização, prevenindo, assim, que os alimentos estejam em risco de contaminação constante. A UAN E foi classificada como ruim para o referido item, por apresentar falta de controle e de gerenciamento do prazo de validade de alguns insumos alimentares, dos alimentos pré-preparados e 
dos alimentos prontos para o consumo.

Souza et al. (2009) apresentaram irregularidades semelhantes às do presente estudo, além de apresentar ausência na identificação dos gêneros a serem congelados. Dare, Bernardo e Chaud (2018) apresentaram inconformidades por constatar que os equipamentos utilizados na distribuição não possuíam barreiras de proteção contra a contaminação pelo consumidor. Além do mais, a área de recebimento dos vales refeições era no mesmo local em que a refeição era distribuída, bem como não havia um funcionário específico para receber o pagamento.

Outro aspecto importante para controle do tempo de exposição dos alimentos está na adequação da temperatura do equipamento de distribuição. Esta deve ser controlada e deve estar em conformidade com o tipo de alimento a ser conservado: a quente ou a frio (RODRIGUES et al., 2011). Para conservação a quente, os alimentos devem estar em temperatura superior a $60{ }^{\circ} \mathrm{C}$ por, no máximo, 6 (seis) horas e para frio, devem ser submetidos à temperatura de $10^{\circ} \mathrm{C}$, por até 4 (quatro horas) (BRASIL, 2004). A inexistência desse monitoramento e aferição da temperatura dos alimentos, sendo este um ponto fundamental para o controle de qualidade, pode comprometer a qualidade das refeições ofertadas, tornando um ambiente favorável a uma contaminação veiculada por alimentos (SÃO JOSÉ, 2012).

Em consequência a isso, os alimentos, para não perderem sua qualidade, devem ser conservados em temperaturas adequadas e em condições seguras do ponto de vista microbiológico. A carência do controle de temperatura dos alimentos pode interferir no comprometimento da qualidade das preparações ofertadas (SILVA et al., 2015). Com isso, a RDC n²016/2004 preconiza que para conservação a quente, os alimentos devem ser mantidos em temperatura superior a $60^{\circ} \mathrm{C}$ por até 6 horas (BRASIL, 2004).

Para o item documentação e registro, as UAN institucionais A, C, e F e as hospitalares B e D apresentaram todos os documentos obrigatório exigidos pela RDC n 2016/2004, que são Manual de Boas Práticas (MBP) e os Procedimentos Operacionais Padronizados (POP), bem como estes estavam depositados em locais de fácil acesso, resultado esse também relatado por Dare, Bernardo e Chaud (2018). Entretanto, a UAN institucional G não possuía tais documentos no momento da coleta de dados. Souza et al. (2009) também verificaram que as UAN avaliadas não apresentavam os documentos obrigatórios, como o Manual de Boas Práticas (MBP).

Couto et al. (2005) apontam que um dos principais problemas relacionados aos riscos de contaminações nas UAN está associado com a ausência do MBP e dos POP, pois sem esses documentos haverá, consequentemente, problemas na implantação das boas práticas, visto que a sua implantação é imprescindível para garantir a oferta de refeições livres de contaminantes, com garantia de segurança e qualidade em todos os requisitos higiênico-sanitários.
O MBP é um instrumento que facilita a implementação das boas práticas, assegurando a qualidade higiênicosanitária dos alimentos produzidos e/ou servidos. De Paula et al. (2017) ressaltaram a importância da implementação do MBP nas UAN por representar transformações consideráveis na higienização dos equipamentos, utensílios e instalações internas, assim desenvolvendo um crescente cuidado no fluxo de manipulação dos alimentos.

\section{Conclusão}

A maioria das UAN avaliadas apresentou condições higiênico-sanitárias satisfatórias em boa parte dos itens preconizados pela RDC $\mathrm{n}^{\circ} 216 / 2004$. Entretanto, algumas UAN foram classificadas como ruins, podendo gerar riscos à saúde dos comensais.

Para garantir um fornecimento de refeições seguras, do ponto de vista higiênico-sanitário, alguns pontos precisam ser melhorados em algumas UAN, como as edificações e instalações, as matérias-primas, bem como a elaboração e o cumprimento do Manual de Boas Práticas estabelecido pelas normas regulamentadoras que, por sua vez, é obrigatória e deve ser, periodicamente, revisada.

Além disso, é relevante destacar a necessidade na execução de treinamentos sobre boas práticas na fabricação, objetivando a correção de práticas de manipulação errôneas por parte dos manipuladores, possibilitando a diminuição da contaminação alimentar.

\section{Referências}

ALMEIDA, C. P. B. et al. Conceito de risco e princípio da precaução na vigilância à saúde: a vigilância sanitária de alimentos como cenário de prática. Rev Segurança Aliment. Nutr., v.24, n.2, p.153-160, 2017. doi: 10.20396/san.v24i2.8649934.

ABERC - Associação Brasileira das Empresas de Refeição Coletiva. Mercado Real. 2020. Disponível em: <https://www. aberc.com.br/mercadoreal.asp?IDMenu=21 >. Acesso em: 1 maio 2020.

BEZERRA, I. N. et al. Consumo de alimentos fora do lar no Brasil segundo locais de aquisição. Rev. Saúde Pública, v.51, n.15, p.1-9, 2017. doi: 10.1590/s1518-8787.2017051006750

BOFF, J.M.; STRASBURG, V.J. Avaliação da efetividade de boas práticas em serviços de alimentação coletiva em uma capital brasileira. Rev. Saúde, v.44, n.1, p.1-9, 2018. doi: $10.5902 / 2236583425334$

BRASIL. Ministério da Saúde. Agência Nacional de Vigilância Sanitária. Resolução no 275, de 22 de outubro de 2002. Dispõe sobre o regulamento técnico de procedimentos operacionais padronizados aplicados aos estabelecimentos produtores/ industrializadores de alimentos. Diário Oficial da União. 23 out 2002. p. 1-20.

BRASIL. Ministério da Saúde. Agência Nacional de Vigilância Sanitária. Resolução $n^{\circ} 216$, de 15 de setembro de 2004. Dispõe sobre regulamento técnico de boas práticas para serviços de alimentação. Diário Oficial da União. 16 set 2004. p. 1-14.

BRASIL. Ministério da Saúde. Secretaria de Vigilância em Saúde. Departamento de Vigilância das Doenças Transmissíveis. Coordenação Geral de Doenças Transmissíveis. Surtos de doenças 
transmitidas por alimentos no Brasil - Informe 2018. Disponível em: http://portalarquivos2.saude.gov.br/images/pdf/2019/ fevereiro/15/Apresenta----o-Surtos-DTA---Fevereiro-2019.pdf. Acesso em: 13 maio 2020.

COUTO, S. R. M. et al. Diagnóstico higiênico-sanitário de uma Unidade Hoteleira de Produção de Refeições Coletivas. Rev. Hig. Aliment., v.19, n.131, p.15-18, 2005.

DARE, C.; BERNARDO, D.R.D.; CHAUD, D.M.A. Avaliação das condições físicas e higiênico-sanitárias de uma unidade de alimentação e nutrição hospitalar. Saúde (Santa Maria), v.44, n.1, p.1-7, 2018.

DE PAULA, N. F. et al. Manual de Boas Práticas para Bancos de Alimentos: a perspectiva de uma nova construção. Demetra Aliment. Nutr. Saúde, v.12, n.2, p.361-383, 2017. doi: 10.12957/ demetra.2017.22877

GOMES, N.A.A.A.; CAMPOS, M.R.H.; MONEGO, E.T. Aspectos higiênicos-sanitários no processo produtivo dos alimentos em escolas públicas do Estado de Goiás, Brasil. Rev. Nutr., v.25, n.4, p.473-485, 2012. doi: 10.1590/S141552732012000400005

LEÃO, R.C. et al. Ocorrência de enteroparasitos e coliformes termotolerantes nas mãos de manipuladores de alimentos de um hospital de ensino. Cad. Saúde Coletiva, v.26, n.2, p.211215, 2018. doi: 10.1590/1414-462x201800020283

LOCATELI, G.; SILVA, E.F. Avaliação das condições estruturais e higiênico-sanitárias das unidades de alimentação e nutrição de uma instituição hoteleira de Foz do Iguaçu/PR. Nutr. Brasil, v. 16, n. 5, p. 266-271, 2017.

LUND, B. M.; O’BRIEN, S. J. Microbiological safety of food in hospitals and other healthcare settings. J. Hosp. Infection, v.73, p. 109-120, 2009. doi: 10.1016/j.jhin.2009.05.017

MACIEL, S. E. S. et al. Unidades de alimentação e nutrição: Aplicação de check-list e avaliação microbiológica. Rev. Bras. Hig. Sanidade Anima, v.11, n.4, p.399-415, 2017. doi: 10.5935/1981-2965.20170039

MENDES, T. I. L. et al. Condições higiênicas e sanitárias de unidades de alimentação e nutrição de escolas em tempo integral. Rev. Eletr. Acervo Saúde, v.31, p.1-10, 2019. doi: 10.25248/reas.e1150.2019

OLIVEIRA, A.P.P.; LIBOREDO. J.C. Avaliação da estrutura física em unidades de alimentação e nutrição da cidade de Sete Lagos-MG. Rev. Ciênc. Vida, v.6, n.3, p.1-20, 2018.

PITTELKOW, A.; BITELlO, A. R. A higienização de manipuladores de uma unidade de alimentação e nutrição (UAN). Rev. Destaques Acad., v.6, n.3, p.22-27, 2014. doi: 10.31533/ pubvet.v12n10a191.1-9

REIS, H.F; FLÁVIO, E.F; GUIMARÃES, R.S.P. Avaliação das condições higiênico-sanitárias de uma unidade de alimentação e nutrição hospitalar de montes claros, MG. Rev. Montes Claros, v. 17, n.2, p.68-81, 2015.

RODRIGUES, F.A. et al. Identificação de pontos críticos de controle de preparações proteicas, em uma unidade de alimentação e nutrição. Rev. Hig. Aliment., v.25, n.1, p.192-193, 2011.

SACCOL, A.L.F.; STANGARLIN, L.; HECKTHEUER, L.H. Instrumentos de apoio para implantação das boas práticas em empresas alimentícias. Rio de janeiro: Rubio, 2013.

SANTOS, C. M. S. et al. Avaliação da Estrutura Física de uma Unidade de Alimentação Escolar em Aracaju-SE. Rev. Ciênc. (In) Cena, v.2 n.9, p.40-51, 2019.

SÃO JOSÉ, J. F. B. Contaminação microbiológica em serviços de alimentação. Nutrire, v.37, n.1, p.78-92, 2012. doi: 10.4322/ nutrire.2012.007

SILVA, L. C. et al. Boas práticas na manipulação de alimentos em Unidades de Alimentação e Nutrição. Demetra Aliment. Nutr. Saúde, v.10, n.4, p.797-820, 2015. doi: 10.12957/ demetra.2015.16721

SOUZA, C. H. et al. Avaliação das condições higiênicas-sanitárias em uma Unidade de alimentação e Nutrição Hoteleira, na cidade de Timóteo - MG. Rev. Dig. Nutr., v.3, n.4, p.312-329, 2009.

VAZ, D.S.S.; BENNEMANN, R.M. Comportamento alimentar e hábito alimentar: uma revisão. Rev. Uningá Rev., v.20, n.1, p.108$112,2018$. 